\title{
Lesões potencialmente malignas da região bucomaxilofacial
}

\section{Potentially malignant lesions of the maxillofacial region \\ Lesiones potencialmente malignas de la región maxilofacial}

Nívia Castro Binda

ORCID: https://orcid.org/0000-0002-0409-4265

Universidade Federal do Espírito Santo, Brasil

E-mail: nivia_sgp@hotmail.com

Ana Luiza Castro Binda

ORCID: https://orcid.org/0000-0002-6664-2538

Escola São Francisco de Assis, Brasil

E-mail: aninha_sgp@hotmail.com

Rodolfo Alves de Pinho

ORCID: https://orcid.org/0000-0002-2151-6642

Faculdade Vértice, Brasil

E-mail: rodolfoalvesmtp@gmail.com

Matheus Almeida Ramalho

ORCID: https://orcid.org/0000-0002-5607-1313 Universidade Federal de Jataí, Brasil

E-mail: matheusramalho@discente.ufg.br

Gabrielly Carvalho Leão

ORCID: https://orcid.org/0000-0002-4292-6667 Universidade Federal do Pará, Brasil E-mail: gabrielly.leao@ics.ufpa.br

Bruna Peixoto Girard

ORCID: https://orcid.org/0000-0003-0233-666X Centro Universitário Cesmac, Brasil

E-mail: brunapeixoto5@hotmail.com

Raynara Brito Silva

ORCID: https://orcid.org/0000-0002-8002-6192

Universidade Ceuma, Brasil

E-mail: raynara.silva180@gmail.com

Maria Karoline Gomes da Silva

ORCID: https://orcid.org/0000-0001-8786-3332

Centro Universitário Cesmac, Brasil

E-mail: karolinegomes201@gmail.com

Nívia Delamoniky Lima Fernandes

ORCID: https://orcid.org/0000-0001-8439-2117

Centro Universitário Inta, Brasil

E-mail: delamonikynivia@gmail.com

Jefferson Douglas Lima Fernandes

ORCID: https://orcid.org/0000-0002-5231-3813

Federal University of Ceará Campus Sobral, Brasil

E-mail: jefferson.odonto97@gmail.com

Zildenilson da Silva Sousa

ORCID: https://orcid.org/0000-0002-2707-6123 Centro Universitário Maurício de Nassau Fortaleza, Brasil E-mail: zildenilsonsilva@gmail.com

Manuela Silvestre Monteiro

ORCID: https://orcid.org/0000-0002-7888-4243 Centro universitário Cesmac, Brasil

E-mail: manuelasilvestremedicina@gmail.com

Luana Pereira Ibiapina Coêlho

ORCID: https://orcid.org/0000-0002-2054-959X

Universidade Estadual do Maranhão, Brasil

E-mail: luana ibiapina@hotmail.com

Luanni Souto de Albuquerque Barros

ORCID: https://orcid.org/0000-0001-8591-1361

Centro Universitário Cesmac, Brasil

E-mail: luannisouto@gmail.com

Thales Peres Candido Moreira

ORCID: https://orcid.org/0000-0002-0622-3075

Universidade de São Paulo, Brasil E-mail: t.candido@usp.br 


\author{
Alessandra Martinelli Costa \\ ORCID: https://orcid.org/0000-0003-0990-3911 \\ Universidade Federal de Rondonópolis, Brasil \\ E-mail: alessandramartelli72@gmail.com \\ Myra Jurema da Rocha Leão \\ ORCID: https://orcid.org/0000-0003-1782-5239 \\ Santa Casa de Misericórdia de Maceió, Brasil \\ E-mail: myra.jurema.rocha.leao@gmail.com
}

\begin{abstract}
Resumo
O carcinoma epidermóide oral (CEO) é o tipo mais comum de neoplasia maligna entre os tumores de cabeça e pescoço, correspondendo mais de $90 \%$ dos casos e, apesar dos avanços nas medidas terapêuticas, a maioria dos tumores são diagnosticados em estágio tardio da doença, resultando em um prognóstico ruim e baixas taxas de sobrevida. As desordens com potencial de Malignização (DPM) são anormalidades clínicas e histopatológicas, a nível celular, que podem assumir o caráter de tumor maligno, a qualquer tempo, porém podem permanecer estáveis por um considerável tempo. A literatura aponta que cerca de $80 \%$ de dos cânceres de boca são evoluções de DPM. Conhecer as lesões com potencial de malignização se torna importante, para diagnosticar precocemente o câncer de boca, melhorando no prognóstico e nas taxas de sobrevida. Diante do exposto, este estudo possui como objetivo revisar a literatura acerca das lesões com potencial de malignização, abordando suas principais características que possibilitam identificá-las. Para a construção deste artigo foi feito um levantamento bibliográfico nas bases de dados SciVerse Scopus, Scientific Eletronic Library Online (Scielo), Biblioteca Virtual em Saúde (BVS) e U.S. National Library of Medicine (PUBMED). Foram selecionados artigos contemplados entre os anos de 2010 à 2021. Os resultados desta pesquisa revelam existem quatro principais lesões com grande potencial em se tornar câncer de boca, sendo elas leucoplasia, eritroplasia, quielite actínica e líquen plano oral.
\end{abstract}

Palavras-chave: Líquen plano bucal; Leucoplasia bucal; Câncer oral; Eritroplasia.

\begin{abstract}
Oral squamous cell carcinoma (OEC) is the most common type of malignant neoplasm among head and neck tumors, accounting for more than $90 \%$ of cases and, despite advances in therapeutic measures, most tumors are diagnosed at a late stage of the disease, resulting in poor prognosis and low survival rates. Disorders with potential for malignancy (MPD) are clinical and histopathological abnormalities, at the cellular level, which can assume the character of a malignant tumor at any time, but can remain stable for a considerable time. Literature points out that about $80 \%$ of oral cancers are progressions of MPD. Knowing the lesions with potential for malignancy becomes important for early diagnosis of oral cancer, improving prognosis and survival rates. Given the above, this study aims to review the literature on lesions with potential for malignancy, addressing their main characteristics that make it possible to identify them. For the construction of this article, a bibliographic survey was carried out in the SciVerse Scopus, Scientific Electronic Library Online (Scielo), Virtual Health Library (BVS) and U.S. National Library of Medicine (PUBMED) databases. Articles from 2010 to 2021 were selected. The results of this research show that there are four main lesions with great potential to become oral cancer, namely leukoplakia, erythroplasia, actinic kielitis and oral lichen planus.
\end{abstract}

Keywords: Lichen planus oral; Oral leukoplakia; Oral cancer; Erythroplasia.

\title{
Resumen
}

El carcinoma oral de células escamosas (OEC) es el tipo de neoplasia maligna más común entre los tumores de cabeza y cuello, representa más del $90 \%$ de los casos y, a pesar de los avances en las medidas terapéuticas, la mayoría de los tumores se diagnostican en una etapa tardía de la enfermedad, lo que resulta en en mal pronóstico y bajas tasas de supervivencia. Los trastornos con potencial de malignidad (MPD) son anomalías clínicas e histopatológicas, a nivel celular, que pueden asumir el carácter de un tumor maligno en cualquier momento, pero que pueden permanecer estables durante un tiempo considerable. La literatura señala que alrededor del $80 \%$ de los cánceres orales son progresiones de MPD. Conocer las lesiones con potencial de malignidad se vuelve importante para el diagnóstico precoz del cáncer oral, mejorando el pronóstico y las tasas de supervivencia. Dado lo anterior, este estudio tiene como objetivo revisar la literatura sobre lesiones con potencial de malignidad, abordando sus principales características que permiten identificarlas. Para la construcción de este artículo se realizó una encuesta bibliográfica en las bases de datos SciVerse Scopus, Scientific Electronic Library Online (Scielo), Virtual Health Library (BVS) y U.S. National Library of Medicine (PUBMED). Se seleccionaron artículos de 2010 a 2021. Los resultados de esta investigación muestran que existen cuatro lesiones principales con gran potencial de convertirse en cáncer oral, a saber, leucoplasia, eritroplasia, kielitis actínica y liquen plano oral.

Palabras clave: Plano de liquen bucal; Leucoplasia oral; Cáncer oral; Eritroplasia. 


\section{Introdução}

O carcinoma epidermóide oral (CEO) é o tipo mais comum de neoplasia maligna entre os tumores de cabeça e pescoço, correspondendo mais de 90\% dos casos (Metzger et al., 2021). Atualmente o diagnóstico se baseia no exame clínico e histopatológico e, apesar dos avanços nas medidas terapêuticas, a maioria dos tumores são diagnostica em estágio tardio da doença, resultando em um prognóstico ruim e baixas taxas de sobrevida (Mascitti et al., 2018).

O CEO afeta, predominantemente, homens com idade superior a 40 anos (Guzmán $G$ et al., 2015). Os fatores de risco associados são o uso do tabaco, ingestão excessiva de álcool e infecção pelo Papilomavirus humano (HPV) e acomete o assoalho de boca e a língua, mucosa labial, mucosa bucal, o palato duro, área retromolar e gengiva (Chen et al., 2021).

As desordens com potencial de Malignização (DPM) são anormalidades clínicas e histopatológicas, a nível celular, que podem assumir o caráter de tumor maligno, a qualquer tempo, porém podem permanecer estáveis por um considerável tempo (Dantas Da Silveira et al., 2009). A literatura aponta que cerca de $80 \%$ dos cânceres de boca são evoluções de DPM

Conhecer as lesões com potencial de malignização para o câncer de boca se torna importante, a fim de permitir o diagnóstico desta neoplasia, melhorar seu prognóstico e suas taxas de sobrevida. Diante do exposto, este estudo possui como objetivo revisar a literatura acerca das lesões com potencial de malignização para o câncer de boca abordando suas principais características que possibilitam identificá-las.

\section{Metodologia}

Trata-se de uma revisão integrativa de literatura, de caráter qualitativa. A revisão de literatura permite a busca aprofundada dentro de diversos autores e referenciais sobre um tema específico, nesse caso, lesões potencialmente malignas da região bucomaxilofacial (Pereira et al., 2018).

A fim de que haja direcionamento na pesquisa, delineou-se como questão norteadora: "quais lesões da cavidade buxomaxilofacial apresentam potencial de malignização?"

Para a construção deste artigo foi feito um levantamento bibliográfico nas bases de dados SciVerse Scopus, Scientific Eletronic Library Online (Scielo), Biblioteca Virtual em Saúde (BVS) e U.S. National Library of Medicine (PUBMED). Os artigos foram coletados no período de abril a julho de 2021 e contemplados entre os anos de 2010 à 2021, utilizando os seguintes descritores contidos na lista dos Descritores em Ciência da Saúde (DeCS): "Líquen Plano Bucal”, "Leucoplasia Bucal", "Câncer Oral" e "Eritroplasia".

Considerou-se como critério de inclusão os artigos completos disponíveis na íntegra nas bases de dados citadas, nos idiomas inglês e português e relacionadas com o objetivo deste estudo. Os critérios de exclusão foram: artigos incompletos, duplicados, resenhas, resumos e que não possuem relação com o objetivo do presente estudo.

A estratégia de pesquisa se baseou na leitura dos títulos para encontrar estudos que investigassem a temática da pesquisa. Caso atingisse esse primeiro objetivo, posteriormente, os resumos eram lidos e, persistindo na inclusão, era feita a leitura do artigo completo. Quando havia dúvida sobre a inclusão, o artigo era lido por outro autor e, a decisão de inclusão ou exclusão era tomada em consenso.

Com base na revisão de literatura feita nas bases de dados eletrônicas citadas, foram identificados 1590 artigos científicos, sendo que, apenas 25 atenderam aos critérios de inclusão previamente estabelecidos. O fluxograma com detalhamento de todas as etapas de seleção está na Figura 1. 
Figura 1 - Fluxograma de identificação e seleção dos estudos.

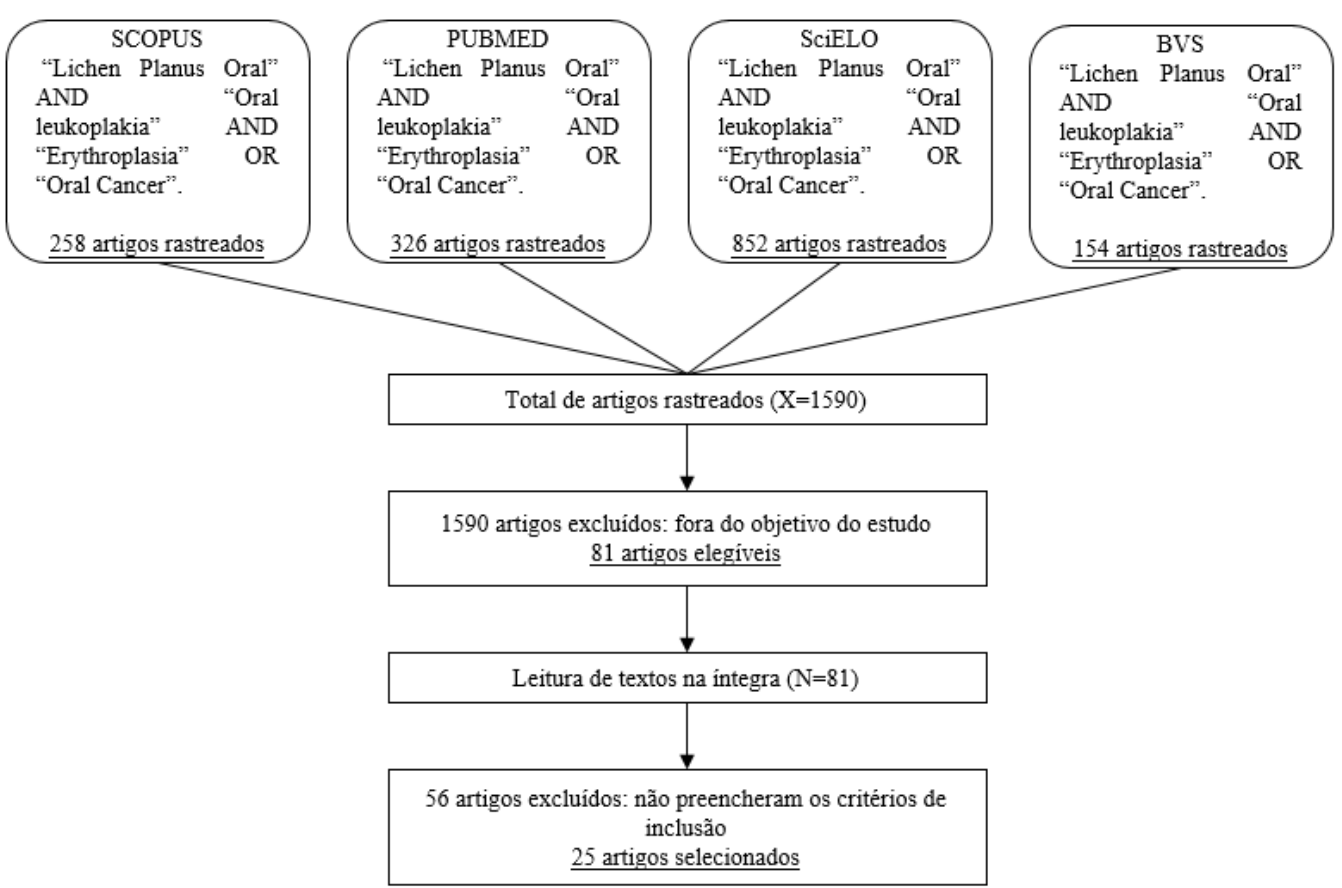

Fonte: Autores (2021).

\section{Resultados e Discussões}

O presente estudo tratou de uma temática atual, preocupante e de urgente atenção, constituindo-se em uma revisão da literatura de pesquisas relacionando lesões com potencial de malignizar. A busca resultou em 25 artigos selecionados que abordaram diretamente o tema proposto, contribuindo para o alcance do objetivo previamente estabelecido.

Os resultados desta pesquisa revelam quatro principais lesões com grande potencial em se tornar neoplasia maligna na região bucomaxilofacial, são elas: leucoplasia, eritroplasia, quielite actínica e líquen plano oral.

\subsection{Leucoplasia}

É definida pela Organização Mundial da Saúde (OMS) como uma lesão hipoplásica não característica de qualquer outra doença. A Leucoplasia é a lesão oral pré-cancerosa mais frequente, representando cerca de $85 \%$ dos casos. O diagnóstico depende da exclusão de outras desordens que possuem as mesmas características clínicas (Ramos et al. 2017).

Geralmente atinge pessoas do sexo masculino (70\%) e acima de 40 anos. A causa da leucoplasia é desconhecida, porém existem várias hipóteses que podem contribuir para o surgimento da lesão, como o uso do tabaco, consumo excessivo de álcool, radiação ultravioleta e microrganismos, como o treponema pallidum, cândida albicans e papilomairus humano (HPV). A Tabela 1 descreve a classificação das leucoplasias quanto às suas características clínicas.

Tabela 1 - Classificação das Leucoplasias.

\begin{tabular}{clc}
\hline Classificação & \multicolumn{1}{c}{ Características Clínicas } & Locais mais frequentes \\
\hline Homogênea & $\begin{array}{l}\text { Lesão uniforme em cor e textura, predominantemente } \\
\text { branca, textura suave, fina ou levemente enrugada. Tipo } \\
\\
\text { mais comum e menos agressivo }\end{array}$ & $\begin{array}{c}\text { Gena jugal } \\
\text { Não-homogênea }\end{array}$ \\
& $\begin{array}{l}\text { Superfície irregular ou verrucosa, podendo possuir áreas } \\
\text { avermelhadas }\end{array}$ & Ventre e borda de língua \\
\hline
\end{tabular}


De acordo com José et al., é possível identificar o estadiamento das leucoplasias, através da extensão e do exame histopatológico, com foco na ausência ou presença de displasia epitelial. O sistema de classificação de tais lesões está descrito na Tabela 2.

Tabela 2 - Sistema de Classificação de Leucoplasia de acordo com Diagnóstico Clínico.

\begin{tabular}{|c|c|c|}
\hline \multicolumn{2}{|c|}{ L = Extensão da Lesão } & \multirow{2}{*}{$\begin{array}{l}\text { I = Histopatológico } \\
\text { Sem displasia }\end{array}$} \\
\hline $\mathrm{L}_{0}$ & $\begin{array}{lll}\text { Sem evidência de } & \text { desão }\end{array}$ & \\
\hline $\mathrm{L}_{1}$ & $\begin{array}{l}\text { Lesões únicas ou } \\
\text { múltiplas juntas } \leqslant 2 \mathrm{~cm}\end{array}$ & $\mathrm{I}_{1} \quad$ Displasia leve \\
\hline $\mathrm{L}_{2}$ & $\begin{array}{l}\text { Lesões únicas ou } \\
\text { múltiplas juntas } \\
\text { medindo entre } 2 \text { à } 4 \mathrm{~cm}\end{array}$ & $\mathrm{I}_{2}$ Displasia moderada \\
\hline $\mathrm{L}_{3}$ & $\begin{array}{l}\text { Lesões únicas ou } \\
\text { múltiplas juntas } \geqslant 4 \mathrm{~cm}\end{array}$ & $\mathrm{I}_{3}$ Displasia grave \\
\hline $\mathrm{L}_{\mathrm{X}}$ & $\begin{array}{l}\text { Lesões com extensão } \\
\text { não especificada }\end{array}$ & IX Não especificado \\
\hline
\end{tabular}

Sendo:

Estágio I $\quad \mathrm{L}_{1} \mathrm{I}_{0}$

Estágio II $\quad \mathrm{L}_{2}$ I0

Estágio III $\quad \mathrm{L}_{3} \mathrm{I}_{0}$ ou L $\mathrm{L}_{2} \mathrm{~L}_{1}$

Estágio IV $\quad \mathrm{L}_{3} \mathrm{I}_{1}$

Fonte: Adaptado de José et al. (2019).

A importância do estadiamento está na possibilidade de estimar o prognóstico e o tratamento mais adequado em uma determinada situação, sendo o estágio I o menos agressivo e o estágio IV o mais grave. O fator mais preditivo para malignização de tais lesões é a presença de displasia nas biopsias (Ramos et al. 2017).

O diagnóstico final para leucoplasia deve ser feito excluindo os possíveis diagnósticos diferenciais, como ceratose traumática, candidíase e lesão por tabaco e confirmado através de exames histopatológicos. Para isso, o protocolo atual para detecção de tal lesão se baseia em uma avaliação clínica criteriosa associado a biopsia direcionada, a fim de identificar uma lesão com potencial de malignização, tratá-la, e excluir a possibilidade de evolução para o câncer de boca (Palmerín-Donoso, Cantero-Macedo \& Tejero-Mas 2020).

\subsection{Eritroplasia}

É definida, pela OMS, como uma eritematosa que não pode ser clinica ou patologicamente diagnosticada como qualquer outra condição. Acomete, predominantemente, pessoas de meia idade ou idosos e não possui predileção por gênero (Tannure et al., 2012).

Tal desordem é menos comum do que a leucoplasia, porém possui maior potencial para malignização. As características clínicas são manifestadas na mucosa como uma mácula ou placa eritematosa bem delimitada, com textura macia 
e geralmente assintomática, possuindo o assoalho de boca e palato mole como sítios mais comuns para o acometimento de tais lesões (Queiroz et al., 2014).

Na maioria dos casos a lesão não possui sintomatologia dolorosa, porém a literatura aborda que há casos em que o paciente apresenta ardência e queimação no local (Maia et al. 2016). O diagnóstico para a eritoplasia, semelhantemente às leucoplasias, deve ser feito excluindo os diagnósticos diferenciais, como carcinoma de células escamosas, doenças autoimunes, doenças infecciosas e lesões traumáticas (Imam, 2013). O tratamento é guiado pelo exame histopatológico e, em casos em que houver algum grau de displasia, deve-se remover as lesões e seguimento ambulatorial para vigilância de possíveis recidivas (Hosni et al. 2009).

\subsection{Quielite Actínica}

É uma desordem considerada pré-maligna, ou seja, com potencial de malignização, comum no lábio inferior que resulta de uma exposição excessiva à radiação ultravioleta da luz solar. Acomete, predominantemente, pessoas do gênero masculino, de pele clara e com idade superior a 40 anos (Arnaud et al., 2014).

Clinicamente a lesão se manifesta pela atrofia da borda do vermelho do lábio inferior, possuindo uma superfície lisa e áreas de manchas claras, crostas, úlceras, edema e erosão. Além disso, há o apagamento da margem entre a zona do vermelho e a pele do lábio (Varela-Centelles et al., 2020), podendo haver sangramento, ressecamento e queimação no local (Gonzaga et al., 2020).

\subsection{Líquen Plano Bucal}

O líquen plano bucal (LPB) é uma doença autoimune que envolve a mucosa e pele, relativamente comum, de caráter crônica e inflamatória. É a desordem dermatológica mais comum na cavidade oral com prevalência de 1 a $2 \%$ da população geral. Cerca de 2 a 3\% de tais lesões evoluem para o câncer de boca. O risco de evolução aumenta quando o portador de LPB está exposto a carcinógenos, como tabaco, álcool, tabaco de mascar e candidíase (Ceita \& Pessoa, 2014).

Tal desordem acomete, preferencialmente, mulheres com idade superior a 30 anos. A Tabela 3 descreve a classificação do LPB de acordo com suas características clínicas.

Tabela 3 - Classificação do Líquen Plano Bucal.

\begin{tabular}{|c|c|c|c|}
\hline Tipo & Considerações clínicas & Sítio de acometimento & Sintomatologia \\
\hline $\begin{array}{l}\text { Forma } \\
\text { Reticular }\end{array}$ & \begin{tabular}{ll} 
- Linhas & \multicolumn{2}{c}{ brancas } \\
entrelaçadas (estrias de \\
Wickham), envoltas por \\
uma borda eritematosa. \\
- Forma mais recorrente.
\end{tabular} & $\begin{array}{l}\text { Região posterior da mucosa jugal } \\
\text { bilateralmente. Podendo acometer, } \\
\text { concomitantemente, a borda lateral } \\
\text { e o dorso da língua, gengiva, } \\
\text { palato, e o vermelho do lábio }\end{array}$ & Assintomático \\
\hline $\begin{array}{l}\text { Forma } \\
\text { Erosiva }\end{array}$ & $\begin{array}{llr}\text { - Áreas atróficas } & \text { e } \\
\text { eritematosas envoltas } & \text { por } \\
\text { estrias irradiadas, com } \\
\text { graus variáveis } & \text { de } \\
\text { ulceração central } & \end{array}$ & Qualquer região da mucosa oral. & $\begin{array}{l}\text { Dor ou queimação no } \\
\text { local }\end{array}$ \\
\hline $\begin{array}{l}\text { Forma } \\
\text { Bolhosa }\end{array}$ & $\begin{array}{l}\text { - Se a lesão for muito grave, } \\
\text { poderá haver a separação } \\
\text { do epitélio e o tecido } \\
\text { conjuntivo, gerando a } \\
\text { forma bolhosa }\end{array}$ & $\begin{array}{l}\text { Mucosa jugal nas regiões posterior } \\
\text { e inferior dos } 2^{\circ} \text { e } 3^{\circ} \text { molares. }\end{array}$ & $\begin{array}{l}\text { Dor no local, } \\
\text { principalmente quando } \\
\text { há rompimento das } \\
\text { bolhas }\end{array}$ \\
\hline
\end{tabular}

Fonte: Autores (2021). 
O diagnóstico do LPB deve ser feito pela avaliação clínica e confirmado pelo exame histopatológico, a fim de descartar outras lesões que possuem manifestações clínicas semelhantes (Leite \& Assay 2019). A forma reticular não necessita, na maioria das vezes, de tratamento. Em contrapartida, a forma erosiva e bolhosa, devem seguir a terapia medicamentosa com corticoesteróides tópicos (Pereda Rojas et al., 2016).

\section{Conclusão}

A partir das trajetórias bibliográficas dos pesquisadores, esse estudo abordou as principais lesões da região bucomaxilofacial que possuem potencial de evoluírem para o câncer de boca, abordando seus conceitos, características clínicas e tratamentos.

Os resultados desta pesquisa revelam quatro principais lesões com grande potencial em se tornar câncer de boca, sendo elas leucoplasia, eritroplasia, quielite actínica e líquen plano oral. O diagnóstico de tais desordens deve ser dado através da avaliação clínica e confirmado pelo exame histopatológico, a fim de descartar outras lesões que possuem manifestações clínicas semelhantes.

Com isso, o levantamento bibliográfico sobre as lesões potencialmente malignas da região bucomaxilofacial se mostra importante, pois envolve estudos retrospectivos que possuem relação com o tema proposto e, ao mesmo, tempo, cria-se a possibilidade futura de novos pesquisadores abordarem o tema, criando novos trabalhos, para que haja melhora na deteç̧ão precoce do câncer de boca, melhorando seu prognóstico e nas taxas de sobrevida desses pacientes.

\section{Referências}

Arnaud, R. R., Soares, M. S. M., Paiva, M. A. F. de, Figueiredo, C. R. L. V. de, Santos, M. G. C. dos, \& Lira, C. C. (2014). Queilite actínica: avaliação histopatológica de 44 casos. Revista de Odontologia Da UNESP, 43(6), 384-389. https://doi.org/10.1590/1807-2577.1038

Ceita, J., \& Pessoa, U. F. (2014). Líquen Plano Oral-Lesão Pré-Maligna?

Chen, X., Zhao, W., Chen, S., \& Yu, D. (2021). Mutation profiles of oral squamous cell carcinoma cells. Advances in Oral and Maxillofacial Surgery, 2, 100026. https://doi.org/https://doi.org/10.1016/j.adoms.2021.100026

Dantas Da Silveira, É. J., Lopes, M. F. F., Silva, L. M. M., Ribeiro, B. F., Lima, K. C., \& Queiroz, L. M. G. (2009). Potentially malignant oral lesions: Clinical and morphological analysis of 205 cases. Jornal Brasileiro de Patologia e Medicina Laboratorial, $45(3)$, 233-238. https://doi.org/10.1590/s167624442009000300008

Gonzaga, A. K. G., Mafra, R. P., da Silva, L. P., de Almeida Freitas, R., de Souza, L. B., \& Pinto, L. P. (2020). Actinic cheilitis: Morphometric parameters and its relationship with the degree of epithelial dysplasia. Acta Histochemica, 122(1), 151452. https://doi.org/https://doi.org/10.1016/j.acthis.2019.151452

Guzmán G, P., Villaseca H, M., Antonio P, L., Araya O, J., Aravena M, P., Cravero P, C., Pino M, P., \& Roa S, J. (2015). Carcinoma epidermoide oral y orofaríngeo: Estudio clínico-patológico. Revista Chilena de Cirugía, 63(3), 250-256. https://doi.org/10.4067/s0718-40262011000300003

Hosni, E. S., et al. (2009). “Oral Erythroplakia and Speckled Leukoplakia: Retrospective Analysis of 13 Cases.” Brazilian Journal of Otorhinolaryngology 75(2):295-99.

Imam, A. (2013). Alterações potencialmente malignas da mucosa oral. Journal of Chemical Information and Modeling, 53(9), 1689-1699.

José, H., Coelho, R., Macedo, C., Antonio, M., \& Costa, N. (2019). Uma análise retrospectiva de lesões malignas e desordens a retrospective analysis of malignant and potentially malignant disorders diagnosed in seven years Resumo Abstract. 46(1), 5-13.

Leite, A. C., \& Assay, N. T. (2019). "Descoberta de Carcinoma de Células Escamosas Em Lesão Previamente Diagnosticada Como Líquen Plano Oral: Malignização Ou Erro de Diagnóstico Inicial?” 8:178-81.

Maia, H. C. de M., et al (2016). “Potentially Malignant Oral Lesions: Clinicopathological Correlations.” Einstein (Sao Paulo, Brazil) 14(1):35-40.

Mascitti, M., Orsini, G., Tosco, V., Monterubbianesi, R., Balercia, A., Putignano, A., Procaccini, M., \& Santarelli, A. (2018). An overview on current noninvasive diagnostic devices in oral oncology. Frontiers in Physiology, 9(OCT), 1-8. https://doi.org/10.3389/fphys.2018.01510

Metzger, K., Moratin, J., Horn, D., Pilz, M., Ristow, O., Hoffmann, J., Freier, K., Engel, M., \& Freudlsperger, C. (2021). Treatment delay in early-stage oral squamous cell carcinoma and its relation to survival. Journal of Cranio-Maxillofacial Surgery, $49(6)$, $462-467$. https://doi.org/https://doi.org/10.1016/j.jcms.2021.02.007

Palmerín-Donoso, A., (2020). “Oral leukoplakia TT Leucoplasia oral.” Atencion primaria 52(1):59-60. 
Research, Society and Development, v. 10, n. 11, e185101119452, 2021

(CC BY 4.0) | ISSN 2525-3409 | DOI: http://dx.doi.org/10.33448/rsd-v10i11.19452

Pereda Rojas, M. E., González Cardona, Y., \& Torres Herrera, L. W. (2016). Actualización sobre liquen plano bucal. Correo Científico Médico, 20(3), 539555.

Pereira, A., Shitsuka, D., Parreira, F., \& Shitsuka, R. (2018). Método Qualitativo, Quantitativo ou Quali-Quanti. In Metodologia da Pesquisa Científica. https://repositorio.ufsm.br/bitstream/handle/1/15824/Lic_Computacao_Metodologia-Pesquisa-Cientifica.pdf?sequence=1.

Queiroz, S. I. M. L., De Medeiros, A. M. C., Silva, J. S. P. Da, \& Silveira, É. J. D. Da. (2014). Clinical and histopathological evaluation and habits associated with the onset of oral leukoplakia and erythroplakia. Jornal Brasileiro de Patologia e Medicina Laboratorial, 50(2), 144-149. https://doi.org/10.5935/16762444.20140008

Ramos, R. T., et al (2017). “Leucoplasia Oral: Conceitos e Repercussões Clínicas.” Revista Brasileira de Odontologia 74(1):51.

Tannure, P. N., Barcelos, R., Carolina, M., \& Carnasciali, G. (2012). |Eritroplasia bucal: aspectos de interesse ao cirurgião-dentista. Revista Brasileira de Pesquisa Em Saúde/Brazilian Journal of Health Research, O(0), 94-97. https://doi.org/10.21722/rbps.v0i0.3414

Varela-Centelles, P., Seoane-Romero, J., García-Pola, M. J., Leira-Feijoo, Y., \& Seoane-Romero, J. M. (2020). Therapeutic approaches for actinic cheilitis: therapeutic efficacy and malignant transformation after treatment. International Journal of Oral and Maxillofacial Surgery, 49(10), 1343-1350. https://doi.org/https://doi.org/10.1016/j.ijom.2020.02.014 